\title{
MiR-25 overexpression inhibits titanium particle-induced osteoclast differentiation via down-regulation of mitochondrial calcium uniporter in vitro
}

Weifan $\mathrm{Hu}^{1,2}$, Yongbo Yu' ${ }^{1}$, Yang Sun ${ }^{2}$, Feng Yuan ${ }^{2}$ and Fengchao Zhao ${ }^{2^{*}}$

\begin{abstract}
Background: Mitochondrial calcium uniporter (MCU) is an important ion channel regulating calcium transport across the mitochondrial membrane. Calcium signaling, particularly via the $\mathrm{Ca}^{2+}$ /NFATc1 pathway, has been identified as an important mediator of the osteoclast differentiation that leads to osteolysis around implants. The present study aimed to investigate whether down-regulation of MCU using microRNA-25 (miR-25) mimics could reduce osteoclast differentiation induced upon exposure to titanium (Ti) particles.
\end{abstract}

Methods: Ti particles were prepared. Osteoclast differentiation of RAW264.7 cells was induced by adding Ti particles and determined by TRAP staining. Calcium oscillation was determined using a dual-wavelength technique. After exposure of the cells in each group to Ti particles or control medium for 5 days, relative MCU and NFATc1 mRNA expression levels were determined by RT-qPCR. MCU and NFATC1 protein expression was determined by western blotting. NFATC1 activation was determined by immunofluorescence staining. Comparisons among multiple groups were conducted using one-way analysis of variance followed by Tukey test, and differences were considered significant if $p<0.05$

Results: MCU expression was reduced in response to miR-25 overexpression during the process of RAW 264.7 cell differentiation induced by Ti particles. Furthermore, osteoclast formation was inhibited, as evidenced by the low amplitude of calcium ion oscillation, reduced NFATc1 activation, and decreased mRNA and protein expression levels of nuclear factor-KB p65 and calmodulin kinases II/IV.

Conclusions: Regulation of MCU expression can impact osteoclast differentiation, and the underlying mechanism likely involves the $\mathrm{Ca}^{2+}$ /NFATC1 signal pathway. Therefore, MCU may be a promising target in the development of new strategies to prevent and treat periprosthetic osteolysis.

Keywords: Mitochondrial calcium uniporter, miR-25, Periprosthetic osteolysis, Osteoclast, Titanium particle

*Correspondence: zhjianzh@vip.sina.com; xzykdxzfc@163.com

2 Department of Orthopedics, The Affiliated Hospital of Xuzhou Medical

University, 99 Huaihai Road, Quanshan District, Xuzhou City, Jiangsu Province 221000, People's Republic of China

Full list of author information is available at the end of the article

\section{Background}

Titanium (Ti) implants are commonly used in artificial joint replacements, and the influence of $\mathrm{Ti}$ on the periprosthetic environment and the subsequent effects on implant fate have been the subject of much research [1-3]. Sundfeldt et al. estimated that $10-15 \%$ of Ti prosthetics used in artificial joint replacement will loosen and 
require replacement within 15-20 years after implantation [4]. This is attributed to the inevitable wear of the materials caused by relative motion of the prosthetic and degradation/degeneration of the biomaterials, which will further generate wear debris particles. Particles from all implant types will induce the differentiation of osteoclasts and accelerate an inflammatory response, processes that eventually lead to periprosthetic osteolysis. The final results are joint loosening and prosthesis failures $[5,6]$.

Signaling mediated via calcium ions $\left(\mathrm{Ca}^{2+}\right)$, an important messenger responsible for cell transduction, is involved in osteoclast differentiation. When osteoclast precursors are continuously stimulated by $\mathrm{Ca}^{2+}$ oscillation, the calcium-binding protein calmodulin undergoes a conformational transition, which further induces activation of its downstream effector proteins the $\mathrm{Ca}^{2+}$ / calmodulin-dependent protein kinases (CaMKs) and calcineurin phosphatase, which is responsible for nuclear translocation of nuclear factor of activated T cells, cytoplasmic 1 (NFATc1), regulation of osteoclast- specific genes, and thus the promotion of osteoclast differentiation [7]. Moreover, Yang et al. demonstrated through down-regulation of regulator of G-protein signaling 10 (RGS10) that $\mathrm{Ca}^{2+}$ oscillations influence the process of osteoclast formation in vitro, resulting in increased bone mass, whereas osteoclast formation can be inhibited by removal of $\mathrm{Ca}^{2+}$ oscillation [8].

Mitochondria are the most important organelles that regulate $\mathrm{Ca}^{2+}$ signals in the cytoplasm [9]. Mitochondria can extract a large amount of $\mathrm{Ca}^{2+}$ from a specific subcellular structural domain and regulate $\mathrm{Ca}^{2+}$ oscillations, thereby controlling $\mathrm{Ca}^{2+}$ signal within cellular solute and affecting cellular functions [10]. Uptake of $\mathrm{Ca}^{2+}$ ions into mitochondria is mainly mediated by mitochondrial calcium uniporter (MCU), a calcium channel with high selectivity that excludes other cytosolic cations [11]. This behavior relies on membrane potential, through which $\mathrm{Ca}^{2+}$ rapidly moves into mitochondrial matrix along the electrochemical gradient, instead of energy supply from ATP hydrolysis as well as accompanied by co-transformation of other ions and molecules [12].

Mammalian MCU consists of MICU1/MICU2, MCUb, and EMRE (essential MCU regulator) subunits. The selective filter of $\mathrm{MCU}$, which is formed by an $\alpha$ helix that combines $\mathrm{Ca}^{2+}$ with acidic amino acid rings, can distinguish $\mathrm{Ca}^{2+}$-binding sites, expose the negatively charged surface of the inter-membrane space, reduce the energy barrier for $\mathrm{Ca}^{2+}$ flux into the mitochondria, and achieve high selectivity for $\mathrm{Ca}^{2+}$ $[11,13,14]$. Through these actions, MCU controls $\mathrm{Ca}^{2+}$ flux across the mitochondrial membrane. When the cytosolic calcium concentration increases, MCU can rapidly sense, absorb and transport $\mathrm{Ca}^{2+}$ to mitochondrial matrix, directly influencing the frequency and amplitude of calcium signals within cytosol [15]. Therefore, the fate of cells can be mediated by suppressing or enhancing MCU levels to regulate cellular calcium signaling [16].

Because MCU is a ruthenium $(\mathrm{Ru})$-responsive channel, $\mathrm{Ru}$ compounds are commonly investigated as inhibitors of MCU that are able to efficiently enter cells [17]. However, $\mathrm{Ru}$ is a retardant for many cations, and thus, it may result in systemic dysfunctions related to these cations [18]. Other forms of MCU regulation have commonly involved posttranscriptional regulations. MicroRNAs (miRNAs) represent an important class of posttranscriptional regulators. Additional research revealed that when cardiac myocytes are exposed to $\mathrm{H}_{2} \mathrm{O}_{2}$, MCU expression is negatively correlated with miR-25 expression, and miR-25 overexpression can reduce mitochondrial $\mathrm{Ca}^{2+}$ overload and inactivate mitochondrial apoptosis, increasing the resistance of cardiac myocytes to oxidative damage [19]. In addition, a bioinformatics prediction algorithm has identified miR-25 as complementary to the $3^{\prime}$ untranslated region (UTR) sequence of $\mathrm{MCU}$, with a $100 \%$ match of the informatics nucleotide at the 1075 site. Another experimental study showed that MCU expression is significantly negatively correlated with miR- 25 expression, confirming that MCU is a direct target of miR-25 [20]. Thus, we chose miR-25 mimics as a potential regulator of MCU expression in the present study (Table 1 ).

Accordingly, we hypothesized that osteoclast differentiation could be mediated via regulation of calcium signaling achieved by inhibiting MCU. To test this hypothesis, we established an osteolysis model based on osteoclast differentiation of RAW 264.7 cells induced by Ti particles. miRNA- 25 mimics were used to reduce MCU expression and thereby diminish mitochondrial $\mathrm{Ca}^{2+}$ oscillations. RAW 264.7 cells were transfected with miRNA-25 mimics, and the effects on MCU expression and $\mathrm{Ti}$ particle-induced osteolysis were investigated. The findings of this study are expected to provide a new strategy and target sites for the prevention and treatment of periprosthetic osteolysis.

Table 1 Experimental design

\begin{tabular}{ll}
\hline Group & Cells and treatments \\
\hline A & RAW264.7+ miR-25 NC \\
B & RAW264.7+ miR-25 mimics \\
C & RAW264.7+ miR-25 NC+Ti particles \\
D & RAW264.7+ miR-25 mimics + Ti particles \\
\hline
\end{tabular}




\section{Methods \\ Materials}

Ti particles were obtained from ThermoFisher Scientific (purity 93\%, diameter $<20 \mu \mathrm{m}$, USA). Phosphate-buffered saline (PBS) was purchased from Hyclone (USA). miR25 mimics and miR-25 negative control (NC) (sequence: sense, AGUCUGGCUCUGUUCACGUUAC; antisense, GUAACGUGAACAGAGCVAGACU) were synthesized by GenePharm (Shanghai, China). Lipofectamine ${ }^{\mathrm{TM}}$ RNAiMAX (Invitrogen 2149333) was obtained from ThermoFisher Scientific. Fura-2 AM (S1052) was purchased from Beyotime Biotechnology (China). The tartrate-resistant acid phosphatase (TRAP) staining kit (387A) was obtained from Sigma-Aldrich (USA). The reverse transcription (RT) kit (MR101-01/02) and qPCR reaction kit (Q711-02) were obtained from Vazyme Biotech Co., Ltd (Nanjing, China). Paraformaldehyde and glycerin were purchased from Sinopharm Chemical Reagent Co., Ltd. (Shanghai, China). Triton X-100, Hoechst 33258, radioimmunoprecipitation assay (RIPA) lysis buffer and polymethylsulfonyl fluoride (PMSF) were purchased from Beyotime Biotechnology. The BCA protein assay kit (BL521A) was obtained from BIOSHARP (USA). The supplier information for primer sequences is presented in Additional file 1: Table S1, and that for antibodies is included in Additional file 2: Table S2.

\section{Preparation of Ti particles}

$\mathrm{Ti}$ particles were first treated to achieve endotoxin removal as described previously [21, 22]. Briefly, Ti particles were baked at $180{ }^{\circ} \mathrm{C}$ for $6 \mathrm{~h}$, then immersed in $75 \%$ ethanol solution, and shaken on a horizontal rotator for $48 \mathrm{~h}$. After centrifugation, the collected Ti particles were re-dispersed in PBS for further sterilization treatment with high temperature and pressure. The Pierce LAL Chromogenic Endotoxin Quantitation Kit (ThermoFisher Scientific) was applied to ensure that endotoxin concentration among Ti particles was less than $0.1 \mathrm{EU} / \mathrm{mL}$.

The final concentration of $\mathrm{Ti}$ particles used to induce osteoclast differentiation in this study was $0.1 \mathrm{mg} / \mathrm{mL}$, in accordance with previous studies [21, 22].

\section{Cell culture and transfection}

RAW 264.7 cells were obtained from the Cell Bank of the Chinese Academy of Sciences (Shanghai, China). Cells in the logarithmic phase of growth were considered most suitable for further experiments. When the cell density reached 40-50\%, cells were transfected using Lipofectamine $^{\mathrm{TM}}$ RNAiMAX (Invitrogen 2149333) according to the manufacturer's instructions. Then the cells were cultured for $48 \mathrm{~h}$ after transfection before use in experiments. Osteoclast differentiation of RAW264.7 cells was induced by adding Ti particles $(0.1 \mathrm{mg} / \mathrm{mL})$ into the culture medium. The experimental groups are outlined in Table 1.

\section{Western blot analysis}

After exposure of the cells in each group to Ti particles or control medium for 5 days, RIPA lysis buffer and PMSF were used to extract cellular protein. The total protein amount was assessed using a BCA protein assay kit, and $30 \mu \mathrm{g}$ total protein was collected from each sample for further analysis. The collected proteins were separated on sodium dodecyl sulfate (SDS)-polyacrylamide gel electrophoresis (PAGE) gels and transferred to membranes (Millipore HATF00010). After blocking with nonfat dried milk for $1 \mathrm{~h}$ at room temperature, the membranes were incubated with primary antibody overnight at $4{ }^{\circ} \mathrm{C}$. The second antibody was then added for $1 \mathrm{~h}$ of incubation at $37{ }^{\circ} \mathrm{C}$. The specific bands were recorded using a chemiluminescence (ECL) detection system (Bio-Ras, ChemiDocXRS+, USA). The relative intensities of the protein bands were analyzed using ImageJ software (National Institutes of Health, USA), using $\beta$-actin as an internal reference.

\section{Determination of calcium oscillation}

After exposure of the cells in each group to $\mathrm{Ti}$ particles or control medium for 24, 72, or 120 h, Fura-2 AM was added to the culture medium for a 45 -min incubation at room temperature. The dual-wavelength technique was applied using a Luminescence Detector (Molecular Devices SpectraMax Rouge ${ }^{\circledR} \mathrm{i} 3$, USA) with excitation wavelengths of 340 and $380 \mathrm{~nm}$. The intensity ratio $(340 / 380 \mathrm{~nm})$ was recorded over time to reflect calcium oscillation.

\section{TRAP staining}

After exposure to $\mathrm{Ti}$ particles or control medium for 5 days, RAW 264.7 cells were fixed and stained according to the instructions of the TRAP staining kit. The samples were observed under an optical microscope (Leica DMI3000B, Germany). Cells that were positively stained and had more than three nuclei were regarded as osteoclasts. Ten areas in each well were photographed for counting of osteoclasts within each field. The counts were repeated three times for each field and averaged.

\section{Immunofluorescence staining}

Cells from each treatment group were fixed for $30 \mathrm{~min}$ in $4 \%$ paraformaldehyde and treated with Triton X-100 $(0.1 \%)$. For staining, the cells were incubated with NFATc1 antibody at $4{ }^{\circ} \mathrm{C}$ overnight, then rinsed with PBS several times, and incubated with second antibody for $1 \mathrm{~h}$ in darkness at $37{ }^{\circ} \mathrm{C}$. More specifically, the cells were fixed before cell membrane permeability $(0.2 \%$ 
tritonX-100/PBS) was enhanced for being more conducive to the binding of NFATc1 antibody into the nucleus. Fluorescent staining is to verify the activation of NFATc1 in the nucleus.

The detailed information for the secondary antibodies used is provided in Additional file 2: Table S2. After staining with Hoechst33258 for $15 \mathrm{~min}$ in the darkness at room temperature, the staining slices were sealed using glycerin. The stained cells were observed using a fluorescence microscope (Olympus, Japan).

\section{Real-time PCR}

After exposure of the cells in each group to Ti particles or control medium for 5 days, the total RNA from each sample was extracted using the Trizol method. Reverse transcription was carried out to obtain cDNA using the RT kit, and then the mRNA expression levels of MCU, NFATc1, CaMKII/IV and nuclear factor (NF)- $\mathrm{kB}$ p65 were determined using the qPCR reaction kit. The results were analyzed using a real-time fluorescent quantitative PCR instrument (LightCycler ${ }^{\circledR}$ 480II, Roche, Switzerland). Differences in the expression levels of target genes among the various groups were analyzed using the $2^{-}$ $\Delta \Delta \mathrm{CT}$ method. The amplification conditions were set as: $94{ }^{\circ} \mathrm{C}$ for $10 \mathrm{~min}$ followed by 40 cycles of $94{ }^{\circ} \mathrm{C}$ for $20 \mathrm{~s}$, $55^{\circ} \mathrm{C}$ for $20 \mathrm{~s}$, and $72{ }^{\circ} \mathrm{C}$ for $20 \mathrm{~s}$. The primers used for reverse transcription were designed and synthesized by Sangon Biotech (Shanghai, China), and the sequences are listed in Additional file 1: Table S1.

\section{Statistical analysis}

All data are presented as mean \pm standard derivation (SD). Statistical analysis was carried out using GraphPad Prism 8 software (GraphPad, USA). Comparisons among multiple groups were conducted using one-way analysis of variance (ANOVA), and then Tukey test was used to identify significant differences between two groups. Unless otherwise noted, differences were considered significant if $p<0.05$.

\section{Results}

Effect of miR-25 on MCU expression, calcium oscillation, and osteoclast formation

To verify that miR-25 expression would lead to downregulation of $\mathrm{MCU}$ expression during the process of osteoclast differentiation, we measured MCU expression in each treatment group. After 5 days in culture with control medium (no Ti particles), cells transfected with miR-25 mimics (group B) showed lower MCU mRNA and protein levels than those transfected with miR-25 NC (group A; $p<0.001$; Fig. 1a-c). After 5 days in culture with medium containing Ti particles, the MCU mRNA level in cells transfected with miR-25 mimics and exposed to Ti particles (group D) was significantly lower than that in cells transfected with miR-25 NC and then exposed to Ti particles (group C; $p<0.0001$; Fig. 1a). The same trend was observed for $\mathrm{MCU}$ protein expression (Fig. 1b, c). These results confirm that overexpression of miR-25 led to reduced MCU expression independent of the induction of osteoclast differentiation.

To examine the effect of MCU down-regulation on $\mathrm{Ca}^{2+}$ oscillations in the cells, we applied Fura-2 AM staining on days 1,3 , and 5 in the different treatment groups. As shown in Fig. $1 \mathrm{~d}, \mathrm{Ca}^{2+}$ oscillation was extremely low in the absence of $\mathrm{Ti}$ particles, whereas $\mathrm{Ca}^{2+}$ oscillation appeared with exposure to Ti particles. Importantly, the amplitude $\mathrm{Ca}^{2+}$ oscillation in cells of group C (miR-25 $\mathrm{NC}+$ Ti particles) was much greater than that in cells of group D (miR-25 mimics + Ti particles), indicating that down-regulation of MCU led to diminished $\mathrm{Ca}^{2+}$ oscillation during osteoclast differentiation.

The effect of MCU down-regulation on Ti particleinduced osteoclast differentiation was investigated by TRAP staining. Upon exposure to $\mathrm{Ti}$ particles, RAW264.7 cells transfected with either miR-25 mimics or NC appeared as multinucleated osteoclasts with positive TRAP staining. However, with fewer and smaller osteoclasts were observed among the cells treated with miR-25 mimics and Ti particles (group D) compared with the cells treated with miR-25 NC and Ti particles (group C; Fig. 2a). The mean numbers of osteoclasts counted in the microscopic fields for groups $C$ and D were $41.7 \pm 5.5$ and $21.3 \pm 4.5$ cells, respectively, indicating a significant $41.2 \%$ reduction in osteoclast formation with miR-25 mimics treatment (Fig. 2b).

\section{Effect of decreased MCU expression on $\mathrm{Ca}^{2+} /$ NFATc1 signaling}

NFATc1 is an important transcription factor in the process of osteoclast differentiation that is activated by $\mathrm{Ca}^{2+}$ oscillation. To examine whether NFATc1 expression changed consistently with the reduced $\mathrm{Ca}^{2+}$ signaling observed after MCU down-regulation by miR-25 mimics treatment, the cells of the different treatment groups were subjected to immunofluorescence staining to detect NFATc1 activation. As shown in Fig. 3a, compared to cells treated with miR-25 NC and Ti particles (group C), those transfected with miR-25 mimics and then exposed to Ti particles (group D) exhibited reduced NFATc1 activation. Counting of NFATc1-positive cells in different microscopic fields yielded totals of $335.0 \pm 53.0$ positive cells in group $C$ and $96.0 \pm 38.6$ positive cells in group D, indicating a $71.3 \%$ reduction in NFATc1 activation with MCU down-regulation $(p<0.01)$. Additionally, real-time PCR analysis showed decreased NFATc1 mRNA expression with MCU down-regulation induced by miR-25 


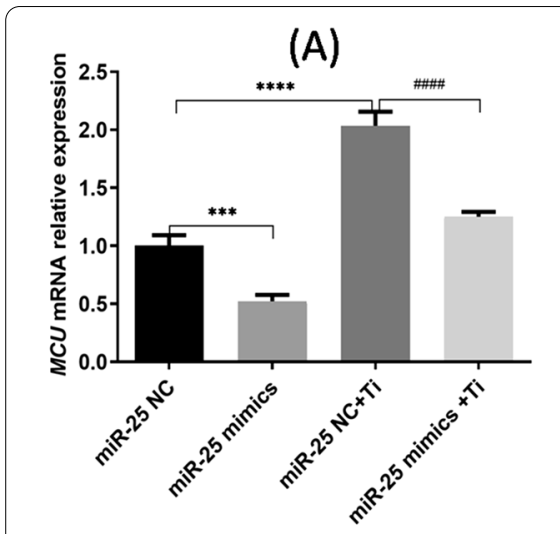

$\mathrm{Ca}^{2+}$ oscillation-24h

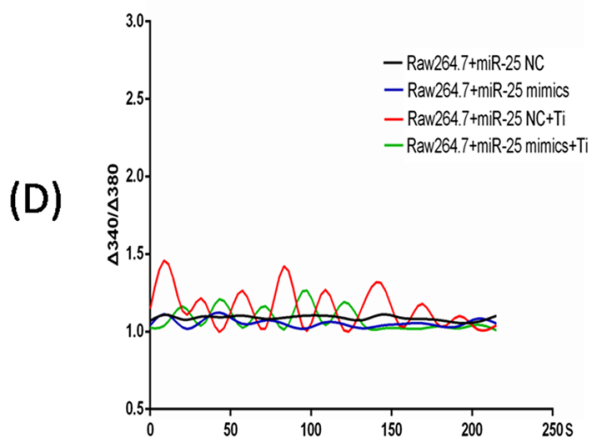

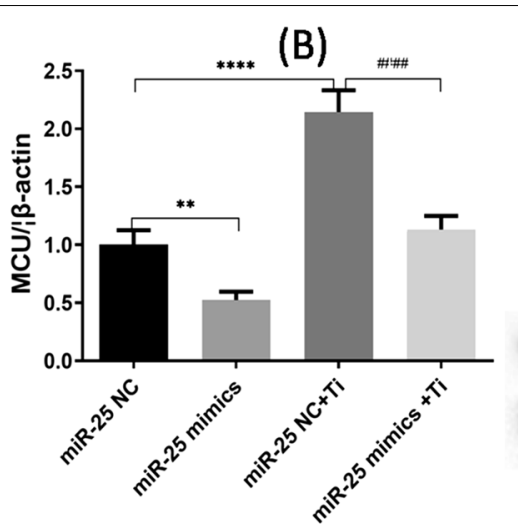

$\mathrm{Ca}^{2+}$ oscillation-72h

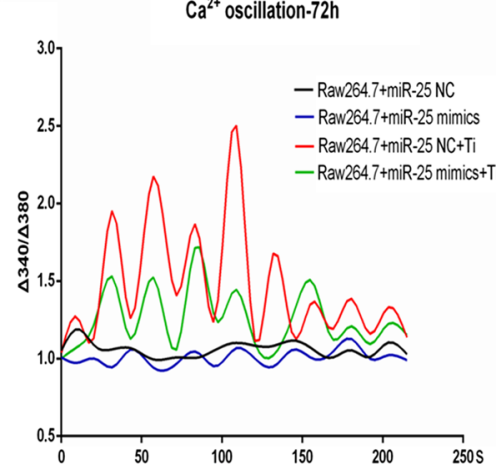

(C)

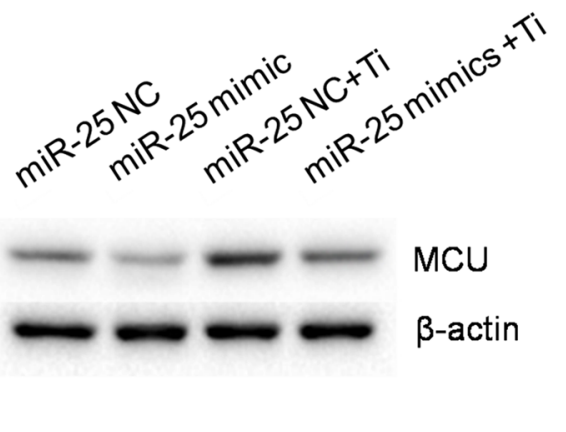

$\mathrm{Ca}^{2+}$ oscillation-120h

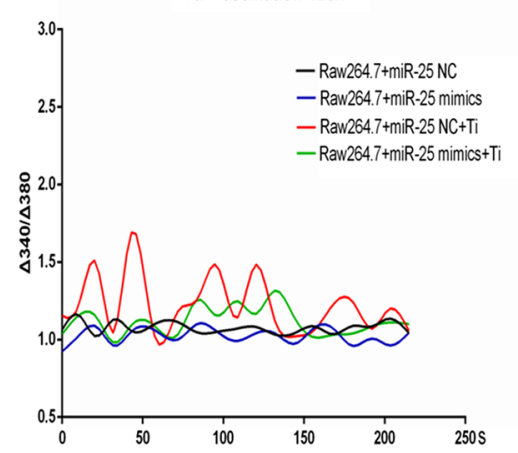

Fig. 1 Reduced MCU expression influences Ca ${ }^{2+}$ oscillations in RAW 264.7 cells. a Relative MCU mRNA expression determined by RT-qPCR in RAW 264.7 cells of the different treatment groups after 5 days. b Western blot analysis of MCU protein expression and c quantified MCU expression relative to $\beta$-actin expression. $\mathbf{d} \mathrm{Ca}^{2+}$ oscillation in RAW 264.7 cells of the different treatment groups at 24,72 , and $120 \mathrm{~h} .{ }^{* *} p<0.01,{ }^{* * *}$ and $\# \#<0.001,{ }^{* * * *}$ and ${ }^{\# \# \#} p<0.0001$

(A)

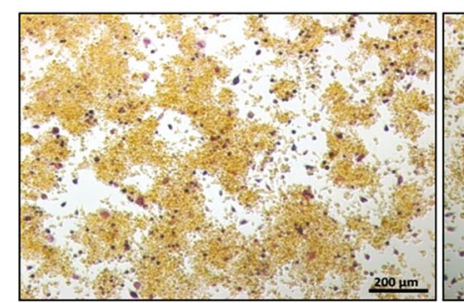

miR-25 NC

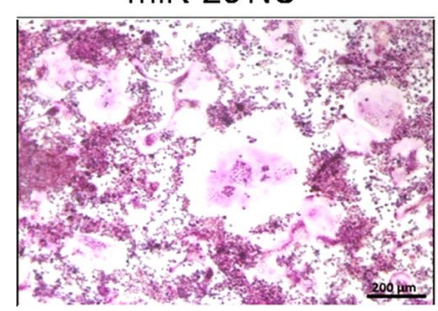

$\mathrm{miR}-25 \mathrm{NC}+\mathrm{Ti}$

\section{(A)}
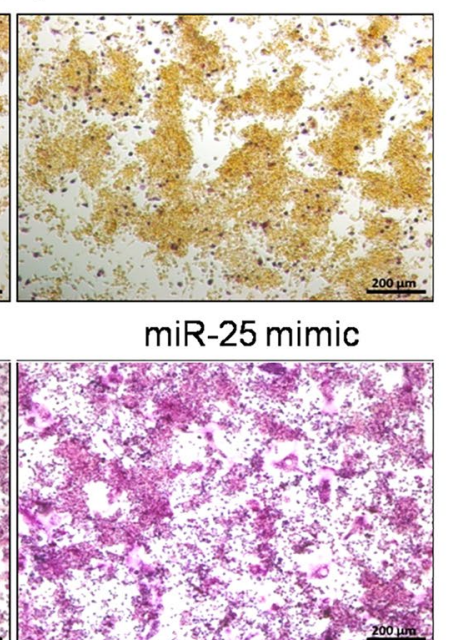

miR-25 mimics $+\mathrm{Ti}$
(B)

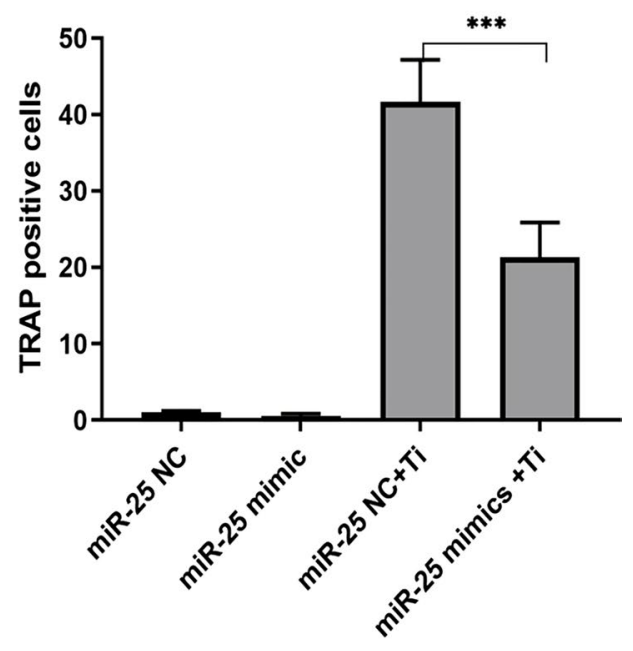

Fig. 2 Down-regulation of MCU reduces osteoclast differentiation. a TRAP staining after exposure of the cells in each group to Ti particles or control medium for 5 days. Scale bars, $200 \mu \mathrm{m}$. b Numbers of osteoclasts counted in microscopic fields. ${ }^{* *} p<0.001$ 


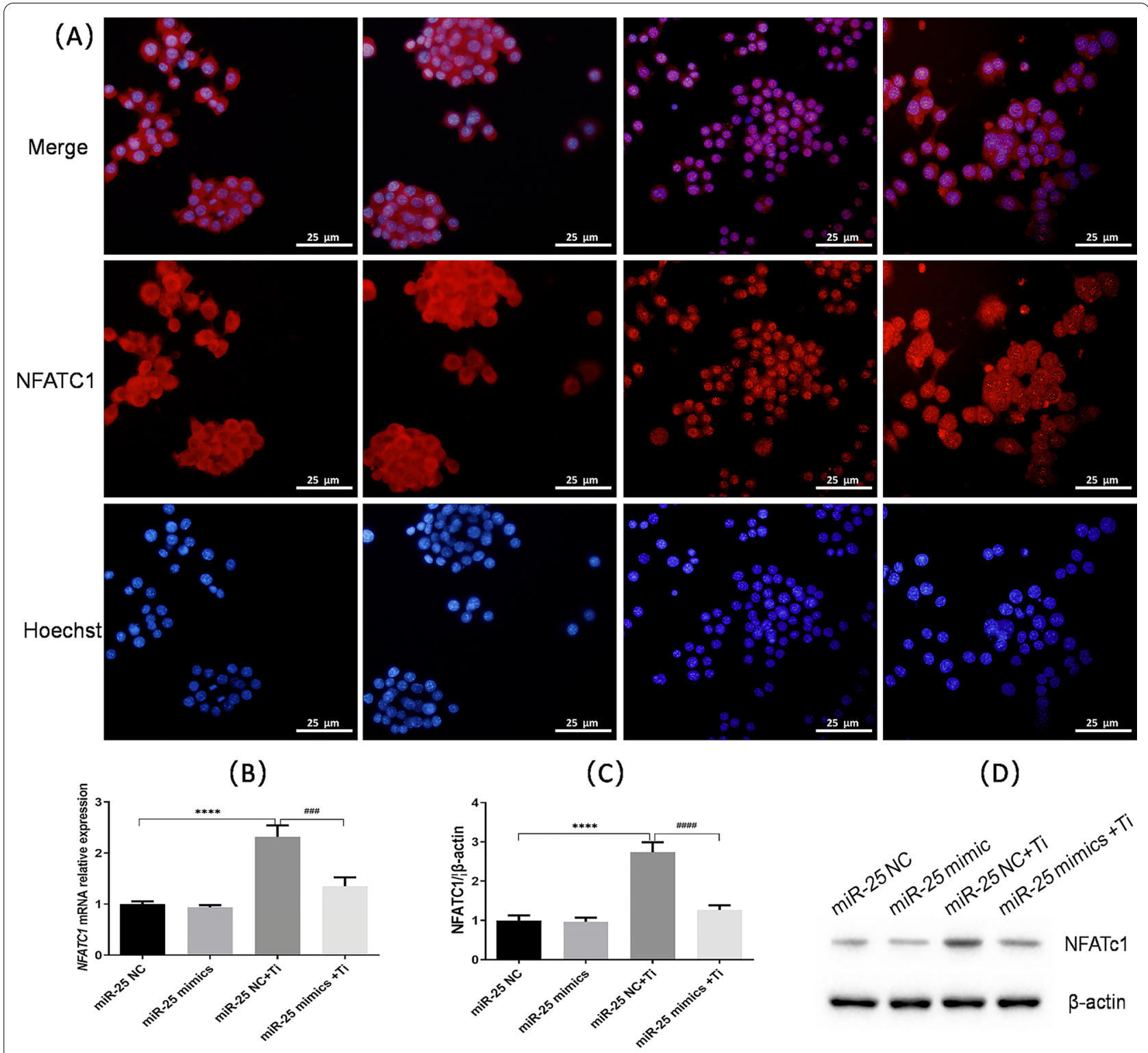

Fig. 3 NFATC1 activation is diminished by reduced MCU expression. a NFATc1 activation determined by immunofluorescence staining of RAW 264.7 cells in the different treatment groups. $\mathbf{b}$ Relative NDATc1 mRNA expression determined by RT-qPCR in the different treatment groups. $\mathbf{c}$ NDATc1 protein expression determined by western blotting, and $\mathbf{d}$ quantitated NDATc1 protein expression, using $\beta$-actin as the internal reference

expression (Fig. 3b). The results for NFATc1 protein expression also showed reduced NFATc1 expression in group D compared with group C ( $p<0.0001$; Fig. 3c, d).

Variation in the intracellular calcium concentration supports the transport of transcription factors from the cytoplasm to the nucleus. During the process of osteoclast differentiation, in addition to NFATc1, NF- $k B$ is another important transcription factor and indicator of inflammatory status. p65 is one of the key subunits of NF- $\mathrm{kB}$. Therefore, we examined NF- $\mathrm{kB}$ expression in cells of the different treatment groups. Compared to cells in group $\mathrm{C}$ (miR-25 $\mathrm{NC}+\mathrm{Ti})$, those in group $\mathrm{D}$ (miR-25 mimics $+\mathrm{Ti}$ ) showed significantly reduced levels of $\mathrm{p} 65$ mRNA expression $(p<0.0001$, Fig. 4a) and NF-кB p65 protein expression $(p<0.01$, Fig. $4 \mathrm{~d})$.

Considering our data showing that decreased $\mathrm{MCU}$ expression influences $\mathrm{Ca}^{2+}$ oscillation and subsequently osteoclast differentiation, we examined whether calmodulin activation of CaMKs was also affected. Indeed, cells in group D (miR-25 mimics + Ti) showed lower mRNA levels of CaMK II and CaMK IV than cells in group C (miR-25 NC+ Ti; Fig. 4c). Moreover, similar results were 


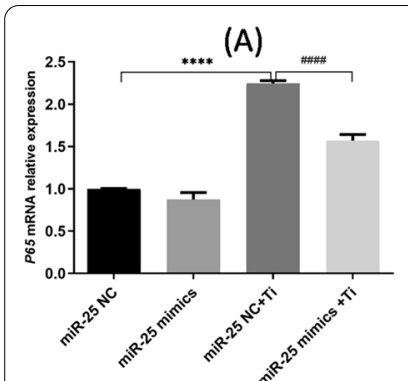

(D)

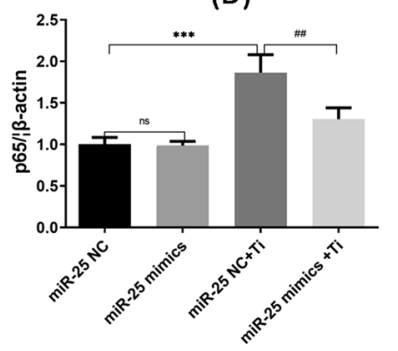

(B)

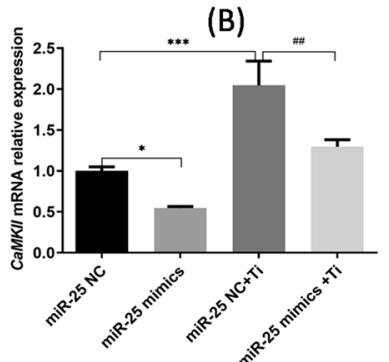

(E)

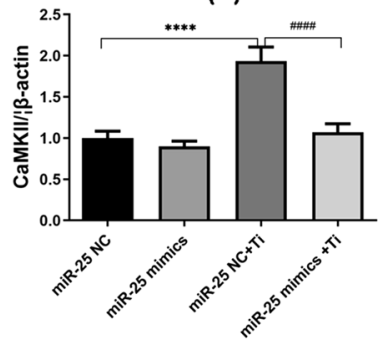

(C)

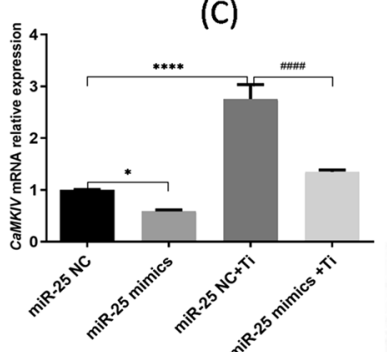

(F)

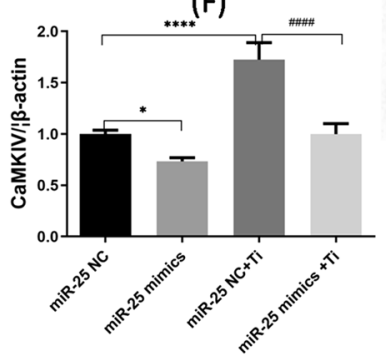

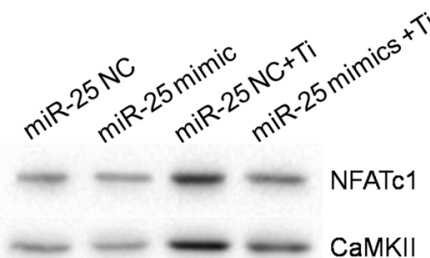

-- CaMKIV

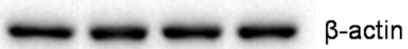

(G)

Fig. 4 Effects of miR-25-mediated MCU down-regulation on the gene and protein expression levels of CaMKs. The relative mRNA expression levels of a NF-KB p65, b CaMK II and c CaMK IV, as determined by RT-PCR. Relative protein expression levels of $\mathbf{d}$ NF-KB p65, e CaMK II and $\mathbf{f}$ CaMK IV, as determined by western blotting and using $\beta$-actin as an internal reference

observed for CaMK II and CaMK IV protein expression (Fig. 4e-g), indicating that miR-25 overexpression downregulated the protein expression levels of these CaMKs.

\section{Discussion}

Artificial joint replacement is an effective treatment strategy for many degenerative joint diseases and arthritis. It is highly successful during the early period postoperation; however, prosthesis loosening during later stages is a major concern. Such loosening is closely related to the increased need for artificial joint replacement. Several risk factors for revision joint surgery have been identified, including advanced age, osteoporosis, high cost, and physicians' experience [23]. Thus, the prevention and treatment of aseptic loosening of prostheses is of great value for prolonging the service life of joint prostheses and reducing revision arthroplasty. Although the exact mechanism of aseptic loosening of prostheses remains to be determined, it is widely accepted that wear debris particles can induce osteoclast differentiation and osteolysis around a prosthesis [24]. As they are the only cells responsible for bone absorption in vivo, the differentiation and regulation of osteoclasts in the periprosthetic environment is of great significance for treating and preventing PPO.

Bisphosphonates have been used clinically to treat osteolysis induced by osteoporosis and some tumors, as they function to inhibit bone adsorption by osteoclasts. However, these drugs have troublesome side effects, such as gastrointestinal adverse reactions and nephrotoxicity, and accurate data supporting their application to prevent and treat osteolysis are lacking $[25,26]$. Two alternatives, osteoprotegerin-Fc (OPG-Fc) and anti-RANKL (receptor activator of NF- $\mathrm{KB}$ ligand) therapy represent a major clinical strategy for inhibiting osteoclast differentiation. However, a wide variety of signaling molecules downstream of the RANKL signaling pathway are involved in complicated regulatory pathways with multiple functions [27]. Although clinical trials have not found an increased risk of bone malignancy or deterioration of metastatic bone tumors, anti-RANKL therapy in mice could cause dysfunction and even deficiency of lymphopoiesis, an effect that may be exacerbated by experimental and spontaneous tumor metastasis and more sensitive to chemical carcinogen [28]. Given the limitations of these therapeutic strategies, the $\mathrm{Ca}^{2+} / \mathrm{NFATc1}$ signaling pathway has become a new target for inhibiting osteoclast differentiation. NFATc1 is known to play a key role in osteoclast differentiation, and with ectopic expression of the NFATc1 gene, bone marrow-derived mesenchymal cells (BMSCs) could differentiate into osteoclasts even without RANKL induction [29]. Moreover, Liu et al. established an in vitro osteolysis model induced by wear debris particles and found that regulation of the $\mathrm{Ca}^{2+} / \mathrm{NFATc1}$ signaling pathway impacted osteoclast generation; specifically, $\mathrm{Ti}$ particle-induced osteoclast differentiation could be suppressed upon inactivation of NFATc1 using 11R-VIVIT peptide [21].

In the process of osteoclast differentiation, when precells are stimulated, calcium channels on plasmatic 
membrane open, allowing the influx of extracellular calcium into cells, resulting in an increased intercellular calcium concentration. To maintain a calcium equilibrium, adjacent channels or organelles such as the endoplasmic reticulum further release calcium to the cytosol, resulting in nonlinear $\mathrm{Ca}^{2+}$ oscillations [30], which directly regulate the process of osteoclast differentiation. MCU plays a great role in this process. Our RT-PCR and western blot analyses confirmed that miR-25 overexpression led to reduced MCU gene and protein expression in RAW264.7 cells, both with and without Ti particle induction of their differentiation into osteoclasts, indicating that miR-25 is a regulator with strong specificity for MCU expression. Meanwhile, with the reduction in MCU levels, $\mathrm{Ca}^{2+}$ oscillations decreased and osteoclast formation was reduced, suggesting that down-regulation of MCU led to altered calcium signaling and, consequently, diminished Ti particle-induced osteoclast differentiation.

In the process of Ti particle-induced osteoclast formation in cells overexpressing miR-25, the mitochondrial $\mathrm{Ca}^{2+}$ influx was decreased and $\mathrm{Ca}^{2+}$ oscillations reduced. Accordingly, activation of NFATc1 was decreased in the absence of continuous stimulation of $\mathrm{Ca}^{2+}$ oscillation, and ultimately, osteoclast activity was diminished. In relation to this process, NF- $\mathrm{KB}$ and NFATc1 have a similar Rel homology domain (RHD) [31], and $\mathrm{Ca}^{2+}$ levels in the cytoplasm are suppressed and cannot be effectively activated to achieve successful nuclear transposition, resulting in reduced mRNA and protein expression of NF- $\mathrm{kB}$ p65 and decreased expression of osteoclast gene products. At the same time, due to their structural similarity, activated NF-kB and NFATc1 can directly interact to form a complex, which allows them to play a synergetic role in promoting transcription [32]. In the present study, $\mathrm{Ca}^{2+}$ oscillations were inhibited upon down-regulation of $\mathrm{MCU}$, preventing this synergetic effect and finally reducing osteoclast differentiation. Of course, the NF- $\mathrm{kB}$ signaling pathway is also an important route for osteoclast differentiation, in which NF- $\mathrm{kB}$ is irreplaceable.

In terms of the $\mathrm{Ca}^{2+} / \mathrm{NFATc} 1$ pathway, CaMK family members (especially CaMK II and CaMK IV) are crucial molecules for the transfer of calcium signals that participate in the differentiation and functions of osteoclasts. $\mathrm{Ca}^{2+}$ oscillation induces a conformation change in calcium-binding proteins, leading to CaMK activation and regulation of osteoclast differentiation via the CaMKCREB route. CaMKs can also regulate osteoclast-specific gene expression in cooperation with NFATc1. On the other hand, CaMKs and c-Fos synergistically act on the promoters of NFATc1 genes and trigger a self-amplification mechanism of NFATc1 [33]. In the present study, with the overexpression of miR-25, CaMK II and CaMK IV levels were reduced, resulting in decreased expression of osteoclast-specific genes, minimizing activation of the self-amplification mechanism of NFATc1 gene expression, and ultimately reducing osteoclast activity. In support of these findings, a recent study on neuronal injury caused by acute ischemia/reperfusion found that by blocking the activation of CaMK II/IKK/NF- $\mathrm{KB}$ signaling, down-regulation of NF- $\mathrm{KB}$ p 65 subunits in mice could increase I/R-induced neuronal death, and up-regulation of CaMK II could increase activation of the NF- $\mathrm{kB}$ upregulating pathway [34]. In consideration of the expression of CaMK and NF- $\mathrm{kB}$ observed in our study, we speculate that interaction of CaMKs and NF- $\mathrm{kB}$ may be another regulatory pathway for osteoclast differentiation.

In this study, transfection with miR-25 mimics decreased MCU expression in RAW 264.7 cells during the process of osteoclast differentiation induced by Ti particles, while also weakening $\mathrm{Ca}^{2+}$ oscillation and reducing NFATc1 activation and NF- $\mathrm{kB}$ expression. Furthermore, miR-25 expression also decreased CaMK (downstream signaling molecules) levels, inhibited osteoclast activity, and restricted osteoclast formation. These data provide experimental evidence for the theory that osteoclast differentiation can be inhibited through the $\mathrm{Ca}^{2+} /$ NFATc1 signaling pathway. During this process, overload of intracellular calcium appeared due to the lack of MCU-mediated regulation of intracellular $\mathrm{Ca}^{2+}$, resulting in functional disorder and apoptosis of osteoclasts and causing osteoclast absorptive dysfunction. Further studies of the underlying mechanisms of these effects are warranted in support of the development of therapeutic strategies targeting MCU.

\section{Conclusion}

In summary, we explored the effect of MCU expression on osteoclast formation. The data obtained in this study confirm that MCU is involved in the process of PPO induction by $\mathrm{Ti}$ particles. Transcription mediated by miR-25 can effectively act on MCU, inhibiting osteoclast differentiation possibly via the $\mathrm{Ca}^{2+} / \mathrm{NFATc1}$ signaling pathway. However, this route for inhibiting osteoclast differentiation might also influence other cellular processes mediated by $\mathrm{Ca}^{2+}$ signaling, and thus, further studies are needed to determine the safety of this approach. Our analysis of $\mathrm{miR} 25 / \mathrm{MCU} / \mathrm{Ca}^{2+} / \mathrm{NFATc} 1$ interaction and its roles in osteoclast differentiation may provide a foundation for the future development of therapies to prevent and/or treat wear particle-induced PPO.

\section{Abbreviations}

MCU: Mitochondrial calcium uniporter; NFATC1: Nuclear factor of activated T cells, cytoplasmic 1; CaMKs: $\mathrm{Ca}^{2+} /$ calmodulin-dependent protein kinases; RANKL: Receptor activator of NF-KB ligand; OPG: Osteoprotegerin; PPO: Periprosthetic osteolysis. 


\section{Supplementary Information}

The online version contains supplementary material available at https://doi. org/10.1186/s13018-022-03030-7.

Additional file 1: Table S1. Sequences of primers used in RT-PCR.

Additional file 2: Table S2. Details of antibodies used in this study.

\section{Acknowledgements}

Not applicable.

\section{Authors' contributions}

WH: Conceptualization; Data curation; Formal analysis; Writing —original draft. YY: Supervision; Validation. YS: Investigation; Methodology; Software. FY: Supervision: Validation. FZ: Project administration; Supervision; Writing review and editing. All authors read and approved the final manuscript.

\section{Funding}

No funding was received for conducting this study.

\section{Availability of data and materials}

The datasets used and/or analysed during the current study are available from the corresponding author on reasonable request.

\section{Declarations}

Ethics approval and consent to participate

Not applicable.

\section{Consent for publication}

Not applicable.

\section{Competing interests}

The authors declare that they have no competing interests.

\section{Author details}

${ }^{1}$ Department of Orthopedics, The People's Hospital of Jiawang District of Xuzhou, Xuzhou 221000, People's Republic of China. ${ }^{2}$ Department of Orthopedics, The Affiliated Hospital of Xuzhou Medical University, 99 Huaihai Road, Quanshan District, Xuzhou City, Jiangsu Province 221000, People's Republic of China.

Received: 29 July 2021 Accepted: 18 February 2022

Published online: 03 March 2022

\section{References}

1. Levack AE, Cyphert EL, Bostrom MP, Hernandez CJ, von Recum HA, Carli $A V$. Current options and emerging biomaterials for periprosthetic joint infection. Curr Rheumatol Rep. 2018;20:33.

2. Ghimire A, Song J. Anti-periprosthetic infection strategies: from implant surface topographical engineering to smart drug-releasing coatings. ACS Appl Mater Interfaces. 2021;13:20921-37.

3. Siverino C, Freitag L, Arens D, Styger U, Richards RG, Moriarty TF, et al. Titanium wear particles exacerbate $\mathrm{S}$. epidermidis-induced implant-related osteolysis and decrease efficacy of antibiotic therapy. Microorganisms. 2021:9:1945.

4. Sundfeldt M, Carlsson L, Johansson C, Thomsen P, Gretzer C. Aseptic loosening, not only a question of wear: a review of different theories. Acta Orthop. 2009:77:177-97.

5. Gallo J, Goodman SB, Konttinen YT, Wimmer MA, Holinka M. Osteolysis around total knee arthroplasty: a review of pathogenetic mechanisms. Acta Biomater. 2013;9:8046-58.

6. Goodman SB, Gallo J. Periprosthetic osteolysis: mechanisms, prevention and treatment. J Clin Med. 2019;8:2091.

7. Ikeda K, Takeshita S. The role of osteoclast differentiation and function in skeletal homeostasis. J Biochem. 2016;159:1-8.
8. Yang S, Li YP. RGS10-null mutation impairs osteoclast differentiation resulting from the loss of $\left[\mathrm{Ca}^{2+}\right]$ i oscillation regulation. Genes Dev. 2007:21:1803-16.

9. De Stefani D, Rizzuto R, Pozzan T. Enjoy the trip: calcium in mitochondria back and forth. Annu Rev Biochem. 2016:85:161-92.

10. Rizzuto R, De Stefani D, Raffaello A, Mammucari C. Mitochondria as sensors and regulators of calcium signalling. Nat Rev Mol Cell Biol. 2012;13:566-78.

11. Baradaran $R$, Wang $C$, Siliciano AF, Long SB. Cryo-EM structures of fungal and metazoan mitochondrial calcium uniporters. Nature. 2018;559:580-4.

12. Boitier E, Rea R, Duchen M. Mitochondria exert a negative feedback on the propagation of intracellular Ca waves in rat cortical astrocytes. J Cell Biol. 1999;145:795-808.

13. Yoo J, Wu M, Yin Y, Herzik MA, Lander GC, Lee S-Y. Cryo-EM structure of a mitochondrial calcium uniporter. Science. 2018;361:506-11.

14. Nguyen NX, Armache J-P, Lee C, Yang Y, Zeng W, Mootha VK, et al. Cryo-EM structure of a fungal mitochondrial calcium uniporter. Nature. 2018;559:570-4.

15. Kamer KJ, Mootha VK. The molecular era of the mitochondrial calcium uniporter. Nat Rev Mol Cell Biol. 2015;16:545-53.

16. Cui C, Yang J, Fu L, Wang M, Wang X. Progress in understanding mitochondrial calcium uniporter complex-mediated calcium signalling: a potential target for cancer treatment. Br J Pharmacol. 2019;176:1190-205.

17. Nemani N, Shanmughapriya S, Madesh M. Molecular regulation of MCU: implications in physiology and disease. Cell Calcium. 2018;74:86-93.

18. Woods J, Lovett J, Lai B, Harris H, Wilson J. Redox stability controls the cellular uptake and activity of ruthenium-based inhibitors of the mitochondrial calcium uniporter (MCU). Angew Chem Int Ed. 2020;59:6482-91.

19. Pan L, Huang B-J, Ma X-E, Wang S-Y, Feng J, Lv F, et al. MiR-25 protects cardiomyocytes against oxidative damage by targeting the mitochondrial calcium uniporter. Int J Mol Sci. 2015:16:5420-33.

20. Marchi S, Lupini L, Patergnani S, Rimessi A, Missiroli S, Bonora M, et al. Downregulation of the mitochondrial calcium uniporter by cancerrelated miR-25. Curr Biol. 2013;23:58-63.

21. Liu FX, Zhu ZA, Mao YQ, Liu M, Tang TT, Qiu SJ. Inhibition of titanium particle-induced osteoclastogenesis through inactivation of NFATc1 by VIVIT peptide. Biomaterials. 2009;30:1756-62.

22. Lee SS, Woo CH, Chang JD, Kim JH. Roles of Rac and cytosolic phospholipase A2 in the intracellular signalling in response to titanium particles. Cell Signal. 2003:15:339-45.

23. Khatod M, Cafri G, Inacio MCS, Schepps AL, Paxton EW, Bini SA. Revision total hip arthoplasty: factors associated with re-revision surgery. J Bone Jt Surg Am Vol. 2015;97:359-66.

24. Zhang L, Yang Y, Liao Z, Liu Q, Lei X, Li M, et al. Genetic and pharmacological activation of Hedgehog signaling inhibits osteoclastogenesis and attenuates titanium particle-induced osteolysis partly through suppressing the JNK/c-Fos-NFATc1 cascade. Theranostics. 2020;10:6638-60.

25. Lozano-Calderon SA, Colman MW, Raskin KA, Hornicek FJ, Gebhardt M. Use of bisphosphonates in orthopedic surgery: pearls and pitfalls. Orthop Clin North Am. 2014;45:403-16.

26. Wilkinson JM. The use of bisphosphonates to meet orthopaedic challenges. Bone. 2020;137:115443.

27. Park-Min K-H. Mechanisms involved in normal and pathological osteoclastogenesis. Cell Mol Life Sci. 2018;75:2519-28.

28. Tanaka S, Nakamura K, Takahasi N, Suda T. Role of RANKL in physiological and pathological bone resorption and therapeutics targeting the RANKLRANK signaling system. Immunol Rev. 2006;208:30-49.

29. Takayanagi $H$, Sunhwa $K$, Koga $T$, Nishina $H$, Isshiki M, Yoshida $H$, et al. Induction and activation of the transcription factor NFATC1 (NFAT2) integrate RANKL signaling in terminal differentiation of osteoclasts. Dev Cell. 2003;3:889-901.

30. Fan M, Zhang J, Tsai C-W, Orlando BJ, Rodriguez M, Xu Y, et al. Structure and mechanism of the mitochondrial $\mathrm{Ca}^{2+}$ uniporter holocomplex. Nature. 2020:582:129-33.

31. Zhu L, Song S, Pi Y, Yu Y, She W, Ye H, et al. Cumulated $\mathrm{Ca}^{2+}$ spike duration underlies $\mathrm{Ca}^{2+}$ oscillation frequency-regulated NF B transcriptional activity. J Cell Sci. 2011;124:2591-601.

32. Liu Q, Chen Y, Auger-Messier M, Molkentin JD. Interaction between NFkB and NFAT coordinates cardiac hypertrophy and pathological remodeling. Circ Res. 2012;110:1077-86 
33. Ye J, Das S, Roy A, Wei W, Huang H, Lorenz-Guertin JM, et al. Ischemic injury-induced CaMKIII and CaMKIly confer neuroprotection through the NF-KB signaling pathway. Mol Neurobiol. 2018;56:2123-36.

34. Sato K, Suematsu A, Nakashima T, Takemoto-Kimura S, Aoki K, Morishita Y, et al. Regulation of osteoclast differentiation and function by the CaMKCREB pathway. Nat Med. 2006;12:1410-6.

\section{Publisher's Note}

Springer Nature remains neutral with regard to jurisdictional claims in published maps and institutional affiliations.

- fast, convenient online submission

- thorough peer review by experienced researchers in your field

- rapid publication on acceptance

- support for research data, including large and complex data types

- gold Open Access which fosters wider collaboration and increased citations

- maximum visibility for your research: over 100M website views per year

At BMC, research is always in progress.

Learn more biomedcentral.com/submissions 